\title{
DE LO MÍTICO A LO PROFANO: CHAC MOOL COMO MONSTRUO
}

\author{
Tatiana Herrera Ávila
}

\section{(c) $(7) \odot$}

Esta obra está bajo una licencia Creative Commons 



\title{
DE LO MÍTICO A LO PROFANO: CHAC MOOL COMO MONSTRUO
}

\author{
FROM MYTH TO THE PROFANE: CHAC MOOL AS A MONSTER
}

Tatiana Herrera Ávila

\begin{abstract}
RESUMEN
Este artículo analiza el cuento "Chac Mool" (Los días enmascarados, 1954) de Carlos Fuentes y la construcción del carácter monstruoso del personaje homónimo, desde la categoría freudiana de lo Unheimlich, esto es lo familiar que ha dejado de serlo. Se abordan específicamente las nociones del doble y la totalidad, de manera que se explica cómo la naturaleza divina y la naturaleza monstruosa, en realidad, se constituyen en caras de la misma moneda. El Chac Mool aparecerá como monstruoso, no solo por su carácter de dios, sino y sobre todo porque para seguir viviendo requiere de la inmolación o el sacrifico del humano, Filiberto, a quien el Chac Mool sustituye, en algún sentido, al final del texto. Se produce así una irrupción de lo mítico en lo profano, y dicha irrupción es, como se verá, monstruosa.

Palabras clave: Chac Mool, Fuentes-Carlos, doble, unheimlich, monstruoso.
\end{abstract}

\begin{abstract}
This article is about Carlos Fuentes' short story "Chac Mool" (1954) and the mounstruous construction of the homonymous character, using Freud's Unheimlich or Uncanny cathegory (this means the familiar which is not familiar anymore (ominous). Specifically, notions like the doppleganger and the completion are central, so that it will be clear that the divine and the mounstruous nature are the same. Chac Mool is mounstruous, not only because it's a god, but also because it needs, Filiberto's sacrifice (a human) for it's own existence, and who will be supplanted by the Chac Mool itself, at the end of the story. That's how an irruption of the sacred into the profane takes place, and this is, by definition, mounstruous.
\end{abstract}

Key words: Chac Mool, Fuentes-Carlos, doppleganger, unheimlich, mounstruous.

Whatsoever I've feared has come to life

Whatsoever I fought off became my life. Just when everyday seemed to greet me with a smile,

Sunspots have faded, now I'm doing time 'cause I fell on black days.

Soundgarden (1994) "Fell on black days".

Lic. Tatiana Herrera Ávila. Universidad de Costa Rica. Profesora de Literatura y Humanidades. Costa Rica. Correo electrónico: tatiana.herrera@ucr.ac.cr

Recepción: 14- 05- 2015

Aceptación: 19- 06- 2015 


\author{
Aquí en la tierra, \\ es un lugar de mucho llanto, \\ lugar donde es bien conocida \\ la amargura y el abatimiento. \\ Un viento como de obsidiana \\ sopla y se desliza sobre nosotros. \\ No hay lugar de bienestar \\ en esta tierra, \\ no hay alegría, no hay felicidad. \\ Códice Florentino
}

\title{
1. Las fuentes antes de la lluvia
}

Llueve y llueve y llueve. A finales de setiembre, en este lado del mundo, eso es absolutamente normal. Desde la perspectiva racionalista y logocéntrica que trajeron los españoles, la lluvia no es un milagro, ni un quehacer divino, sino un fenómeno atmosférico en el cual el agua se precipita de las nubes a la tierra, lo que es necesario para la vida como la conocemos, para la fertilidad y, por lo tanto, para la agricultura. Pero para los pueblos prehispánicos, aquello era obra de los dioses, y hay miles de historias, según la tradición amerindia, donde la lluvia se debe a designios misteriosos y sagrados.

$\mathrm{Y}$ es que en el principio fue el mestizaje. La Conquista dura, cruel, sangrienta, teñida de genocidio. En nombre de la sangre de Cristo se sacrificaron todos los dioses de las mitologías prehispánicas, en honor de la visión de mundo europea, se abrieron las venas de un continente que, aún cinco siglos después, no ha sabido sanar esas heridas. Así el proceso que terminaría por implantar la Modernidad, para Nuestra América, se corresponde con un origen trágico, dejándonos en una ambigüedad cultural, con restos de un pasado que muchas veces queremos olvidar, con una fractura que no nos deja saber con claridad a qué mundo pertenecemos. De ahí la crisis y la tensión que se han manifestado en toda nuestra historia y en la mayoría de nuestra producción artística.

Carlos Fuentes, escritor mexicano nacido en Panamá, da cuenta de dicha problemática en toda su literatura. En relación con esta característica de Fuentes, Oviedo señala que:

\footnotetext{
[...] Su pasión literaria es auténtica, y también lo es su pasión americana, que lo ha movido a representar y analizar la compleja fase de modernización de un país tan antiguo como el suyo, dentro del gran marco de la historia latinoamericana y mundial; es decir, ha compuesto un gran mural, un verdadero friso de la vida pública y privada de nuestro tiempo. (Oviedo, 2002, p. 316)
}

Esto se observa ya desde su primer libro de cuentos Los días enmascarados (1954), donde se encuentra inscrito el cuento "Chac Mool”, que, como se podrá ver, desde su monstruosa discursividad, hace llover la lectura que hoy propongo. Al respecto, Oviedo indica que:

\footnotetext{
El simbolismo del título es significativo porque alude a los cinco días nemontani que, según el calendario azteca, abren un espacio vacío entre el fin de un año y el comienzo del otro, entre el pasado y el futuro -que es también un pasado. Máscaras, dobles, espejos y espejismos son presencias obsesivas en su mundo imaginario; descubrir lo que hay detrás de ellos es lo que guía su esfuerzo creador. (Oviedo, 2002, p. 317)
}

$\mathrm{Y}$ es que esto es clave para el asunto que mueve esta disertación: el Chac Mool como monstruo; pues cabe anticipar que el Chac Mool, se recrea como figura monstruosa en el cuento homónimo, en tanto se construye como doble del protagonista Filiberto, espejismo de un dios y enmascara, además, toda la problemática de la inserción de un México antiguo en el mundo moderno. Esta es la premisa. 
Una vez dicho esto, no hay más que proceder a interpretar la historia de un Chac Mool, ídolo, dios o cautivo que devino en monstruo humano, ${ }^{1}$ siguiendo un tránsito de lo divino a lo profano, pero que, a pesar de eso, hoy, tal vez, siga dependiendo de esa lluvia persistente que no abandona estas tierras americanas. Quiero pensar que así es, quiero permitirme pensar que llueve no por las causas atmosféricas ya sabidas, sino porque Chac Mool camina entre nosotros. Asistamos, sin más, a ese pasaje antropomorfizador y monstruoso del Chac Mool, y dejémonos inundar por las palabras de Fuentes.

\section{El cuento de la lluvia}

El cuento "Chac Mool” se inicia, dejándole saber al lector que Filiberto, un burócrata de cuarenta años ha muerto ahogado en Acapulco, durante Semana Santa. Un amigo, de quien no se dice el nombre -el narrador, en primera instancia-, cuenta que ha ido a recoger las pertenencias del difunto a esta playa y entre ellas descubre un diario. En adelante, se lee la narración de dicho diario, mediante la cual se sabe de los sucesos que llevaron a Filiberto a la muerte. Es así como el lector se entera de que Filiberto ha perdido su trabajo como burócrata en alguna entidad estatal (la Secretaría) y que la causa de esto y su debacle, en todo sentido, incluso de su muerte, es el Chac Mool, un ídolo que le comprara a un mercader, ya que él gustaba de coleccionar ídolos y cacharros.

Se explica, entonces, que el Chac Mool es una especie de dios de la lluvia, que no debe ser confundido con Tlaloc el dios de la lluvia azteca. Una vez que Filiberto tiene a Chac Mool empiezan a ocurrir desgracias en su vida. Primero, Filiberto pone al Chac Mool en el sótano. En ese momento, se rompen las tuberías, y el sótano se inunda. Luego, en las noches se escuchan aullidos y gritos extrañísimos, que resultan, como se indica más adelante en el cuento, provenir de gatos y perros que el Chac Mool se comía, en un proceso de animación, hasta que termina por cobrar vida. En este proceso le va creciendo al Chac Mool un musgo que Filiberto raspaba con mucha paciencia.

Pero el asunto se va volviendo más complicado para Filiberto y cada vez más siniestro, pues el Chac Mool lo va sacando de su casa (al mejor estilo de la cortaziana "Casa tomada"), única herencia de su familia; una casa grande, de estilo porfiriano, lúgubre hoy en día, pero que daba cuenta de un pasado más propicio en la vida de Filiberto. El Chac Mool se viste con su ropa, lo va volviendo loco y se va apropiando de su vida. Chac Mool provoca, además, que Filiberto sea despedido, lo agrede y lo hace su prisionero, su esclavo. Conforme este proceso va ocurriendo, la casa va adquiriendo un olor a sangre y a putrefacción. Hay acá un juego de poder, donde el que creía que era el dominante termina por ser el dominado, como él mismo lo confiesa en su diario. Filiberto, incluso, debe traerle baldes de agua al Chac Mool para mantenerlo húmedo. Y es que el Chac Mool se ponía más agresivo cuando no llovía, esto porque sin la lluvia, se convertiría de nuevo en piedra y volvería a ser inanimado.

Filiberto descubre que Chac Mool ha ido en un proceso de degradación, que ha caído en tentaciones humanas y que hace correrías nocturnas para ir de cacería a conseguir los gatos y perros que se come. Es ahí cuando decide huir a Acapulco, para liberarse de la opresión del Chac Mool y esperar a que este muera antes de que lo mate a él. Sin embargo, Chac Mool, de alguna manera que no se hace patente en el relato, lo alcanza y Filiberto muere ahogado, hecho que posiblemente Filiberto anticipara, en tanto su pasaje es solo de ida. 
Cuando el amigo regresa a la casa de Filiberto, lo que encuentra en esta es un remedo de dios, un indio amarillo, vestido en bata, con los labios mal pintados. Este manifiesta que lo sabe todo y da la orden de que pongan el féretro en el sótano. Se produce así la sustitución de un cuerpo por otro. El Chac Mool está vivo, y es el dueño de la casa y de la vida de Filiberto. Filiberto está muerto, ahogado y, por ello, inundado de agua, y es confinado al sótano. La única conclusión posible que puede concebir el lector es que Filiberto debía morir para que el Chac Mool viviera.

La crítica no ha sido indiferente a este texto, y lo interesante que aflora cuando se hace una revisión de esta es que hay, principalmente, dos líneas interpretativas totalmente opuestas. Por un lado, están las que sostienen que el cuento es un llamado a México para que olvide las viejas supersticiones prehispánicas y mire hacia la promesa de la modernidad, y se hallan aquellas que, más bien, perciben en el texto una convocatoria a recuperar esa herencia tan olvidada entre las candilejas de la vida occidental moderna.

Entre las primeras, los argumentos se centran en que el texto se construye como una parodia de un cuento de horror que termina por burlarse del posible retorno a la adoración de los dioses antiguos (Gottardi, 2013, p. 174). Incluso se llega a señalar que el hablar de estos dioses y de tal herencia es una impostación en Fuentes (Guzmán, 1972, pp. 78-79) y por ello no puede evitar la burla.

Por el contrario, las segundas destacan que el dialogismo y la ambigüiedad presentes en el texto constituyen una evidencia de la tensión existente entre ese México moderno que ha olvidado sus raíces culturales y el pasado antiguo al que habría que recuperar (Camacho-Quiroz, 2011).

Dicha polémica, si bien escapa, relativamente, a lo que atañe a este análisis, resulta más que interesante en la medida en que pone en la palestra uno de los temas que atraviesan la producción de Fuentes, el mestizaje, como marca indeleble de un continente; una marca que, a veces, puede ser una bendición o una maldición. Es en este panorama confuso, en ese clarooscuro donde Fuentes encontró tierra fértil para sus escritos. Y digo relativamente, porque no obstante el interés de este texto es lo monstruoso en "Chac Mool", y terciar en la polémica parece inútil por tanto el texto permite ambas lecturas; tal monstruosidad -sin duda- se ve potenciada por esa tensión de la que Fuentes da cuenta, en el sentido de que puede apuntarse ese México que se debate entre la modernidad y el pasado prehispánico como lo que permite la caracterización monstruosa de Chac Mool. Pero vamos por partes.

El resto de la crítica se avoca a discutir si el cuento es fantástico o gótico (Gottardi, 2013, p. 174), lo cual solo pone de manifiesto, una vez más la tensión que venía comentando, pues el hablar de cuento gótico en América Latina, resulta tan ajeno como hablar de Chac Mool para el México moderno. No obstante, varios autores, Adriana Gordillo de la Universidad de Minnesota (2014) y Antonio Alcalá González (2011) de la Universidad Autónoma de México, entre otros, sostienen esta tesis. Más allá de coincidir o no con dichas posturas, estos trabajos nos interesan en la medida en que destacan la presencia del doble en el cuento, factor central para nuestra lectura como coadyuvante de la construcción de lo monstruoso; pero estos, más bien, se han focalizado en destacar al Chac Mool como elemento fantástico y hasta gótico.

\section{De lluvias y monstruos}

Nos es útil recurrir en este momento, para abordar el elemento monstruoso en "Chac Mool", a Freud y a su artículo "Unheimlich", donde recoge varias definiciones de lo Ominoso para poder desarrollar este tema clave en el psicoanálisis. Nos dice que lo Ominoso, como la palabra 
en alemán lo indica, es aquello que viene de lo familiar, pero que al mismo tiempo no lo es. También, Unheimlich se define como aquello que no es hogareño, no es familiar, no es doméstico. Es el opuesto de heimlich, íntimo. Así lo Unheimlich consiste en lo desconocido, o que debió permanecer desconocido: "[...] lo ominoso es aquella verdad de lo terrorífico que se remonta a lo consabido de antiguo, a lo familiar desde hace largo tiempo" (Freud, 2003, p. 227).

Freud se basa en los estudios de Jentsch para estudiar el fenómeno de lo Ominoso y lo primero que menciona es la movilidad de cosas inertes, la vida de objetos inanimados, como productores de lo Ominoso:

\begin{abstract}
Escribe Jentsch: «uno de los artificios más infalibles para producir efectos ominosos en el cuento literario consiste en dejar al lector en la incertidumbre sobre si una figura determinada que tiene ante sí es una persona o un autómata, y de tal suerte, además, que esa incertidumbre no ocupe el centro de su atención». (Freud, 2003, p. 221)
\end{abstract}

En este sentido, Freud encontró el ejemplo perfecto para lo ominoso en el cuento "El Hombre de Arena", de E. T. A. Hoffman, donde el personaje Nathaniel se enamora perdidamente de una autómata llamada Olimpia sin saber que ella no está viva:

\begin{abstract}
Esta observación [ver cita anterior], sin duda correcta, vale sobre todo para el cuento El Hombre de Arena incluido en las Piezas nocturnas de Hoffman; de él la figura de la muñeca Olimpia ha sido tomada por Offenbach para el primer acto de su ópera «Los cuentos de Hoffman». (Freud, 2003, p. 227)
\end{abstract}

En "El hombre de arena", Olimpia es un ser vivo ante los ojos de Nathaniel. Él la percibe como una bella mujer de la que se enamora. Baila con ella, la besa, la observa desde su ventana, habla con ella y le responde. Pero Nathaniel cae en cuenta de que Olimpia es una muñeca cuando su creador, el profesor Spalanzani, y Coppola, quien lo había ayudado a construirla, se la están peleando. Él interviene desesperado y los ojos de Olimpia caen al piso, mientras Coppola se la lleva entre los gritos del profesor, quien insistía en que ella era su mejor autómata: "Nathaniel se había quedado petrificado; acaba de darse cuenta de que el lívido rostro de cera de Olimpia tenía, en vez de ojos, dos negros agujeros, que era un muñeco sin vida." (Hoffman, 1977, p. 163).

Otro hecho típicamente ominoso, según Freud, es la presencia del Doble, y esto nos interesará en la medida en que el Chac Mool se comportará como un doble para Filiberto, en tanto le roba su vida.

El concepto del doble se encuentra presente en casi todos los grupos humanos, a partir de los espejos y de los gemelos. Borges en su Libro de los seres imaginarios, lo reseña muy someramente, haciendo básicamente una serie de citas de su aparición en la literatura (Poe, Yeats, Dostoievski y Hawthorne, entre otros) y en algunas mitologías. Llama la atención la imagen que Escocia tiene del doble: lo llaman "Fetch, porque viene a buscar (fetch) a los hombres para llevarlos a la muerte" (Borges, 1999, p. 84). Nos interesa esta nota del escritor argentino, porque apunta dos hechos importantes: por un lado el carácter ominoso del doble, y su relación con lo mítico y primitivo del hombre. Ambas aristas están intrínsecamente relacionadas, pues las dos tienen que ver con sus capacidades terroríficas. De igual manera, ya señalaba Borges lo monstruoso de las reduplicaciones, como los espejos. El que el doble o fetch lleve los hombres a la muerte, o sea su condición de psicopompo, se corresponde con lo que ocurre en el cuento de Fuentes, pues de hecho el Chac Mool conduce a Filiberto a su muerte (Fuentes, 2013, párr. 21), como se analizará más adelante.

Y es que el sentimiento de lo ominoso, siniestro, monstruoso o unheimlich, surge en el acercamiento a lo real. Lo real es aquello tan traumático que no puede ser dicho, es 
insoportable y está fuera del lenguaje. El primer trauma, fundante de la vida psíquica, es el nacimiento. Al salir del vientre materno, el niño pierde la perfección y completud. Por esto, el niño desea a la madre (metáfora de la totalidad), por lo cual se establece la relación diádica del Edipo entre el niño y la madre. En este momento, aparece el Padre o Significante Paterno, se interpone entre el niño y la madre, e introduce la Ley primordial, la prohibición del incesto, con lo cual el niño es inscrito en la Cultura, sujeto al Lenguaje. Ahora que el niño ha sido sujetado al lenguaje, se le prohíbe el acceso a la madre, por lo cual se inaugura la falta. Esta carencia de la madre es lo que genera el deseo. El deseo siempre es deseo por la madre, ella es el objeto que falta, esa es la carencia: se carece de la madre, se desea a la madre. Este deseo es el motor de la vida psíquica del sujeto. Somos sujetos por la falta de la madre, objeto primordial que deseamos y esta carencia es lo que mantiene el aparato psíquico del sujeto en movimiento. La vida psíquica es el constante movimiento del deseo por el lenguaje. El lenguaje es el registro simbólico lacaniano (Baudes de Moresco, 1995, p. 47). Cuando no hay deseo, es porque no hay falta, estamos en el dominio de lo real, pues para que haya lenguaje y estemos en lo simbólico debe haber falta, debe haber deseo.

De ahí que lo ominoso, que es otro nombre de lo monstruoso, ubica al sujeto fuera de lo simbólico, y a menudo, ante este enfrentamiento el sujeto zozobra, y muere. Como se verá tal es el caso de Heriberto, pues:

\footnotetext{
[...] el doble es una creación del Yo que remite a la muerte. Porque si, originalmente, es una tentativa de negación de la muerte, él queda como su soporte y lo agita continuamente cuando se produce [...] Finalmente, permaneciendo como portador de una significación mortífera, él es enemigo del Yo, y cuando se presenta al Yo el doble aparece más bien como espectro. (Blumel, 1988, p. 46)
}

De manera que obligatoriamente el doble se presenta como un espectro, concepto que está relacionado en su etimología con lo especular y nos remite al narcisismo primario, en un primer momento. Por ello, la imagen especular está ineludiblemente relacionada con la muerte. Pero además, paradójicamente al intentarse que el doble prolongue la vida, a la vez remite a la muerte. "[...] cuando el doble se exhibe, cuando el Yo se ve al Yo, él no puede hacer sin que se perfile en su horizonte un espectro, la muerte." (Blumel, 1988, p. 46).

Así, al producirse una imagen especular del Yo, que se percibe como otro Yo, y que surge con el objetivo de perpetuar el Yo ad æternitatem, más allá de la muerte, por metonimia, entra en consideración la muerte. "[...] con la superación de esta fase [el narcisismo primario] cambia el signo del doble: de un seguro de supervivencia, pasa a ser el ominoso anunciador de la muerte." (Freud, 2003, p. 235).

Es decir que la sola imagen especular del doble que nace para tratar de escapar de la muerte, remitirá justamente por ello al espectro de la muerte, en una remisión también especular.

\section{Ecce monstrum: Chac Mool}

El fenómeno de lo ominoso posee, como se puede ver, muchas aristas o facetas, ya que es una categoría muy compleja, y nos hemos referido únicamente a aquellas que se manifiestan de manera más que elocuente en el Chac Mool, por cuestiones de claridad y de especificidad. El texto es el que llama a las categorías teóricas, y no al contrario.

Así las cosas, puede afirmarse, de hecho, que todo el cuento de Fuentes es ominoso u "unheimlich", y no solo el Chac Mool, como tal. Veamos por qué. Desde el principio, al describir la situación del cadáver de Filiberto, se presenta en el texto la atmósfera de lo 
unheimlich. Sabemos en la primera frase que Filiberto murió ahogado en Semana Santa: "Hace poco tiempo, Filiberto murió ahogado en Acapulco. Sucedió en Semana Santa." (Fuentes, 2013, párr. 1).

En primera instancia, esto no tendría nada de particular, si no fuera porque como en la literatura nada es gratuito, la mención de que la muerte del personaje ocurrió en Semana Santa debe ser interpretada. Como se sabe, Semana Santa es la festividad más importante de la Cristiandad, según la cual se celebra, la muerte y resurrección de Dios hecho hombre, o del hijo de Dios, Cristo. Esto nos da la clave del texto, pues ocurrirá en el texto, la mezcla entre lo divino y lo profano, y si se quiere una inversión de lo que se celebra en la Semana Mayor: el Chac Mool que resucita en humano, Filiberto, en lugar de la resurrección de un hombre Jesús a su condición de Hijo de Dios. Esta inversión y confusión entre lo divino y lo profano es "unheimlich" porque mezcla dos ámbitos que en principio están separados, y transforma al dios en ser humano. Al respecto, el mismo cuento de Fuentes nos da la pista para hacer esta interpretación:

Pero un Dios al que no le basta que se sacrifiquen por él, sino que incluso va a que le arranquen el corazón, ¡caramba, jaque mate a Huitzilopotchli! El cristianismo, en su sentido cálido, sangriento, de sacrificio y liturgia, se vuelve una prolongación natural y novedosa de la religión indígena. (Fuentes, 2013, párr. 4)

Y es que además, la sola mención de la muerte resulta ominosa, como apunta Freud (Freud, 2003, pp. 226 y 241), y en el texto, es aún más ominoso si se quiere, pues no es solo que Filiberto haya muerto, es incluso, la triste condición la que se encuentra el cadáver, ya que se elimina la vida del cuerpo y este pasa a otro estado que para los vivos resulta aterrador, en tanto no hay psique en el sujeto y ya no le podemos reconocer:

\footnotetext{
Filiberto esperaba, muy pálido en su caja, a que saliera el camión matutino de la terminal, y pasó acompañado de huacales y fardos, la primera noche de su nueva vida. Cuando llegué, temprano, a vigilar el embarque del féretro, Filiberto estaba bajo un túmulo de cocos. (Fuentes, 2013, párr. 1)
}

Como puede verse, hay aquí una situación de deshumanización literalmente, donde Filiberto es una cosa más, algo que fue humano y estuvo vivo, pero ya no. Y es más, como se indica en el cuento: "a ver si no le habíamos echado sal al viaje" (Fuentes, 2013, párr. 1), porque llevar un muerto puede ser de mala suerte (el mal de ojo y semejantes, ostentan siempre carácter ominoso). He ahí, lo ominoso, si partimos de lo que explicara Freud.

Otro elemento que caracteriza al Chac Mool como "unheimlich", y que es el anverso de lo anterior, constituye el factor ya mencionado de la animación de lo inanimado, el cual se hace más que patente en el cuento de Fuentes, pues el Chac Mool es en sí una figura que inicia siendo un objeto inanimado y que, luego, adquiere vida:

\footnotetext{
Hoy, domingo, aproveché para ir a la Lagunilla. Encontré el Chac Mool en la tienducha que me señaló Pepe. Es una pieza preciosa, de tamaño natural, y aunque el marchante asegura su originalidad, lo dudo. La piedra es corriente, pero ello no aminora la elegancia de la postura o lo macizo del bloque. El desleal vendedor le ha embarrado salsa de tomate en la barriga para convencer a los turistas de la autenticidad sangrienta de la escultura. El traslado a la casa me costó más que la adquisición. Pero ya está aquí, por el momento en el sótano mientras reorganizo mi cuarto de trofeos a fin de darle cabida. Estas figuras necesitan sol, vertical y fogoso: ese fue su elemento y condición. Pierde mucho en la oscuridad del sótano, como simple bulto agónico, y su mueca parece reprocharnos que le niegue la luz. El comerciante tenía un foco exactamente vertical a la escultura, que recortaba todas las aristas, y le daba una expresión más amable a mi Chac Mool. Habría que seguir su ejemplo. (Fuentes, 2013, párr. 8)
}

Como se ve, es una estatua de tamaño natural y hecho de piedra, un objeto que Filiberto puede poseer, es $\mathrm{Su}$ Chac Mool. No obstante, a lo largo del cuento, el Chac Mool irá 
tomando vida, primero emitiendo lamentos terribles en las noches (párr. 10), luego cambiando su material: la piedra se va cubriendo de un musgo verde (párr. 13), lo que hace al Chac Mool, grotesco, según palabras del propio Filiberto, y de una alergia. Luego se cubre de vello en los brazos, (párr. 15), y se va volviendo poco a poco, en piel dorada, como la de un Dios (párr. 18), hasta que por último adquiere movimiento, y se anima por completo:

Allí estaba Chac Mool, erguido, sonriente, ocre, con su barriga encarnada. Me paralizaban los dos
ojillos, casi bizcos, muy pegados a la nariz triangular. Los dientes inferiores, mordiendo el labio superior,
inmóviles; sólo el brillo del casquetón cuadrado sobre la cabeza anormalmente voluminosa, delataba vida.
Chac Mool avanzó hacia la cama; entonces empezó a llover. (Fuentes, 2013, párr. 21)

Tras de todo tiene un olor nauseabundo, "extrahumano" (párr. 23), lo que colabora a su carácter ominoso, sin duda.

Por otro lado, Chac Mool se presenta, como Doble de Filiberto, pues poco a poco se va a adueñando de la vida de este. Primero se encontraba en el sótano, luego duerme en la cama de Filiberto y este tiene que dormir en la sala, luego se pone la ropa de Filiberto, y este se ve obligado a doblegarse, no perdamos de vista esta palabra, donde se implica que Filiberto se ha doblado, en el Chac Mool y que este lo domina.

El que el Doble, como señalábamos, sea portador de la muerte es, indudablemente, clave en el cuento (psicopompo, como antes vimos que señalara Borges), pues aunque Filiberto intenta huir de la situación monstruosa en la que se encontraba, termina por morir ahogado, y esto aparentemente era inevitable. Filiberto se ha ido a Acapulco sin comprar un pasaje de regreso y a altas horas de la noche se va a nadar mar adentro, donde perece.

Y es que tal y como señala Blumel, el doble es el enemigo del yo y aparece en un inicio como espectro.

\footnotetext{
Tan terrible como su risilla -horrorosamente distinta a cualquier risa de hombre o animal- fue la bofetada que me dio, con ese brazo cargado de brazaletes pesados. Debo reconocerlo: soy su prisionero. (Fuentes, 2013, párr. 23)
}

Lo más monstruoso del cuento de Fuentes, es que este espectro de risilla que no es de humano ni animal, que tiene un olor extrahumano (de ahí su carácter de espectro), el Chac Mool, termina por tomar el lugar de Filiberto, y Filiberto muere. Pero más allá, Chac Mool ya convertido en una figura humana que los demás perciben, confina el cuerpo de Filiberto al sótano, donde había estado él al principio, se produce así el intercambio, y la suplantación del Doble, que es precisamente lo que lo hace ominoso.

Adicionalmente, un factor que se suma a la construcción del carácter ominoso del Chac Mool es que Filiberto lo coloca, no más lo consigue, en el sótano, a pesar de que sabe que le hace daño al Chac Mool:

\footnotetext{
Estas figuras necesitan sol, vertical y fogoso; es fue su elemento y condición. Pierde mucho en la oscuridad del sótano, como simple bulto agónico, y su mueca parece reprocharme que le niegue la luz. (Fuentes, 2013, párr. 7)
}

El sótano, necesariamente, es metáfora del vientre materno, en tanto oscuro y profundo, e interno, pero además del inframundo, y la ida al inframundo, al igual que el regreso al vientre materno, es unheimlich por excelencia, esto por cuanto se pierde la carencia, y constituye, por ello, un acercamiento a lo Real, en términos freudianos y lacanianos.

En este sentido, es exacto afirmar que Chac Mool es ominoso, y responde literalmente a esto, lo familiar que retorna como extraño, es, si se quiere ese pasado prehispánico del que 
ya no sabemos nada, y que en la modernidad impuesta en América, resulta monstruoso, precisamente por haber sido familiar, por haber sido nuestro, sin serlo ya.

\section{Postlluvia. (Dios hecho hombre: de la modernidad y otros demonios)}

Ahora bien, el carácter monstruoso de Chac Mool queda más que demostrado, incluso el propio Filiberto señala que: "Ya debería estar acostumbrado al Chac Mool, pero hace poco, en la oscuridad, me topé con él en la escalera, sentí sus brazos helados, las escamas de su piel renovada, y quise gritar." (Fuentes, 2013, párr. 25).

Pero, cabe entonces preguntarse: ¿qué función cumple esto? Para responder dicha pregunta, se hace necesario, a mi gusto, retomar la cuestión que mencionáramos al principio sobre la problemática del pasado prehispánico. El Chac Mool vuelve a la vida, pero es un dios venido a menos, un remedo de hombre:

\footnotetext{
Apareció un indio amarillo, en bata de casa, con bufanda. Su aspecto no podía ser más repulsivo; despedía un olor a loción barata; su cara, polveada, quería cubrir las arrugas; tenía la boca embarrada de lápiz labial mal aplicado, y el pelo daba la impresión de estar teñido. (Fuentes, 2013, párr. 31)
}

Filiberto muere ahogado por la presencia del Chac Mool, es decir por la presencia de una herencia cultural no reconocida ni comprendida, mientras que Chac Mool, degradado, hace un tránsito de lo mítico (ese pasado prehispánico) hacia lo profano, cae en las tentaciones humanas, dice Filiberto, y termina revestido de toda la superficialidad y de la artificialidad de la modernidad occidental.

\section{Notas}

1. El Chac Mool es un personaje bastante ambiguo, pues no queda claro, a pesar de lo que el mismo Fuentes explica en su cuento, ni siquiera para los estudiosos, si el Chac Mool era una divinidad azteca o maya, o si quiera si era una divinidad. En Chichen Itzá, se encuentra en uno de los templos principales, la estatua que coincide con la descripción que da Carlos Fuentes, del ídolo de piedra, cuyo vientre serviría, se presume, para sacrificios humanos. Si así fuera, Chac Mool es la piedra sacrificial, solamente. Incluso, Mary Miller (1993, p. 60), ya en 1985, demostró que el Chac Mool de la Mesoamérica Posclásica se deriva de las imágenes mayas de cautivos y víctimas de sacrificio (Schele y Freidel, 1990, pp. 477 y 529). Esto entra en correlación con el análisis que se presenta en este artículo, porque a pesar de que el Chac Mool no fuera una divinidad, posee un carácter sacrificial, que, como bien se explica, colabora al elemento "unheimlich". De igual manera, su carácter ambiguo lo hace aún más ominoso, pues se resiste a ser identificado y reconocido.

\section{Bibliografía}

Alcalá-González, A. (2011). La otredad gótica y la figura del doble. Anuario de letras modernas. 15, 115-126. http://www.revistas.unam.mx/index.php/al_modernas/article/ view/31110 [Consulta 12 de junio de 2014].

Baudes de Moresco, M. (1995). Real, Simbólico, Imaginario. Una introducción. Buenos Aires: Paidós.

Borges, J. L. y Guerrero, M. (1999). El libro de los seres imaginarios. Madrid: Alianza Editorial. 
Blumel, E. (1988). La alucinación del doble. Traducciones. Medellín: Fundación Freudiana de Medellín.

Camacho-Quiroz, R. M. (2011). Dialogismo, intertextualidad e ironía en "Chac Mool” de Carlos Fuentes. Colmena. 69, 156-164. http://www.uaemex.mx/plin/colmena/Colmena_69/ Colmenario/Dialogismo_intertextualidad_ironia_Chac_Mool.pdf [Consulta 03 de junio de 2014].

Freud, S. (2003). Lo ominoso. Obras Completas. (Vol. 17). (2 ed.). Buenos Aires: Amorrortu.

Fuentes, C. (2013). Los días enmascarados. Madrid: Editorial Leer-e.

Hoffman, E. T. A. (1977). El hombre de la arena. El Puchero de Oro. San José: Editorial Costa Rica.

Gordillo, A. (2014). Los fantasmas de la casa. Reflexiones sobre el gótico en la obra de Carlos Fuentes. Badebec. 3 (6), 227-253. http://www.badebec.org/badebec_6/sitio/pdf/ dossier_gordillo.pdf [Consulta 2 de junio de 2014].

Gottardi, S. A. (2013). Chac Mool: ¿Horror gótico o imaginación activa? Hipertexto. 17, 169-176. http://portal.utpa.edu/utpa_main/daa_home/coah_home/modern_home/ hipertexto_home/docs/Hiper17Gottardi.pdf [Consulta 03 de junio de 2014].

Guzmán, D. (1972). Carlos Fuentes. New York: Twayne Publishers.

Miller, M. y Taube, K. (1993). An Illustrated Dictionary of The Gods and Symbols of Ancient Mexico and the Maya. Londres: Thames and Hudson.

Oviedo, J. M. (2002). Historia de la literatura latinoamericana 4. De Borges al presente. Madrid: Alianza Editorial.

Schele, L. y Friedel, D. (1990). Una selva de reyes. La asombrosa historia de los antiguos mayas. México: Fondo de Cultura Económica.

Tapia-Rodríguez, J. (1997). Leyenda y misterio de los aztecas. Barcelona: Edicomunicación. 